\title{
Swiss Medical Board oder: «das Kind mit dem Bade ausschütten»
}

Für die Task Force Prostatakarzinom der Schweizerischen Gesellschaft für Urologie:

Franz Recker ${ }^{a}$

Tullio Sulser ${ }^{b}$

a Mitglied des Scientific Committees der European Randomized Study of Screening for Prostate Cancer ERSPC

b Klinik für Urologie UniversitätsSpital Zürich
In einer Zeit, in der in Schweden vorgesehen ist, ein landesweites aktives Prostata-Screening-Programm einzuführen und auch medizinisch-onkologische Gesellschaften wie die European Society for Medical Oncology (ESMO) eine individuelle Vorsorge aufgrund der Langzeitüberlebensdaten nahelegen, hält das Swiss Medical Board unter der Experten-Führung einer Biomedizinischen Ethikerin, eines klinischen Epidemiologen, Gesundheitsökonomen, klinischen Pharmakologen, medizinischen Strafrechtlers, einer Pflegewissenschaftlerin sowie eines Chirurgen den PSA-Test für untauglich. Lediglich bei familiärer Vorbelastung und symptomatischen Patienten wird der Test noch empfohlen. Dabei liegt der Anteil der Mortalität mit familiärer Vorbelastung lediglich bei ca. $15 \%$. Da PSA gerade bei gutartiger symptomatischer Prostatahyperplasie ohne Karzinom erhöht sein kann, liegt sein besonderer Wert ausgerechnet bei der Früherkennung der abgelehnten asymptomatischen Männer. Das absolut ernst zu nehmende, gravierende, aber sinnvoll anzugehende Problem der Überdiagnostik und Übertherapie mit einer Ablehnung der PSA-Früherkennung lösen zu wollen, bedeutet, das Kind mit dem Bade auszuschütten. Man würde nach 12 bis 15 Jahren auf eine

\section{Themenschwerpunkt zur PSA-Diskussion in der SÄZ Nr. 3/2012 \\ Die auch in der SÄZ* publizierte Empfehlung des Swiss Medical Board, auf die Bestimmung des PSA-Wertes zu verzichten, wenn keine Symp- tome und keine familiäre Belastung vorliegen, hat unter Fachleuten eine intensive Diskussion ausgelöst. In dieser Ausgabe nehmen die Profes- soren Franz Recker und Tullio Sulser im Namen der Task Force Prostatakarzinom der Schweizeri- schen Gesellschaft für Urologie zum Bericht des Medical Board Stellung. Weitere Beiträge wer- den in der zweiten Ausgabe des nächsten Jahres (Erscheinungsdatum 18. Januar) als Themen- schwerpunkt publiziert.}

\section{Die Redaktion}

Korrespondenz:

Prof. Dr. med. Franz Recker

Urologische Klinik

Kantonsspital Aarau

CH-5000 Aarau

* Expertenrat des Swiss Medical Board. PSA-Test nicht geeignet zur Früherkennung des Prostatakarzinoms. Schweiz Ärztezeitung. 2011;92(48):1857.
Reduktion der Prostatakrebssterblichkeit von 50\% verzichten, bei Männern unter 60 Jahren sogar von $60 \%$. Umgekehrt muss die Überbehandlung durch die Active Surveillance (kontrollierte Beobachtung) in Zukunft bei ca. einem Drittel der entdeckten Tumore vermieden werden. Dass dies der durchaus gangbare Weg ist, zeigen Daten der Schweizer ERSPC-Studie (European Randomized Study of Screening for Prostate Cancer) mit 30\% und die Schwedische ERSPCStudie mit $40 \%$ kontrolliert beobachteter Männer ohne Therapie.

Es unterlaufen dem CH Medical Board wie auch der U.S. Preventive Services Task Force USPSTF, die die gleiche Empfehlung ausspricht, wesentliche Fehler, die im Folgenden analysiert werden sollen:

\section{Gravierendste Unzulänglichkeiten der PSA-Empfehlung des Swiss Medical Board}

- Es wird nicht nach Lebensalter für das Vorsorgegespräch differenziert. 50-70-Jährige haben noch eine Lebenserwartung von 15-32 Jahren.

- In der Schweiz sterben 4,5\% der Männer am Prostatakarzinom, 14\% der Prostatakarzinom-Todesfälle ereignen sich bis zum 69. Lebensjahr.

- Die rein epidemiologische Beurteilung der Studien ohne interne Qualitätskriterien des Studienablaufs ist ungenügend. (Quebec, Norrköping, Stockholm sind nicht verwertbar.)

- Die zugrunde gelegten Studien PLCO (Prostate, Lung, Colorectal, and Ovarian Cancer Screening Trial, USA: kein Benefit) und ERSPC (Europa: schon nach 9 Jahren geringer Benefit) sind nicht gleichwertig (PLCO hat schwerste methodische Mängel, genügt nicht B1-Kriterien)

- Die beurteilten Studien sind mit 10 Jahren Follow-up zu kurz, um eine Quantifizierung des Benefits zu erlauben. Die beste LangzeitStudie mit 14 Jahren Follow-up und 50\% Mortalitätsreduktion wird vernachlässigt.

- Die amerikanischen Mortalitätsstatistiken (SEER = Surveillance, Epidemiology, and End Results) und der schwedische SPCG-4-Trial (Scandinavian Prostate Cancer Group Study 4) sind unberücksichtigt.
- Der PSA-Test $(<3 \mathrm{ng} / \mathrm{ml})$ übersieht vernünftigerweise irrelevante Tumore. 
- Eine Gesamtbilanz im Jahr 2011 zu unternehmen, ist wegen fehlender Daten unmöglich. U.a. wird die Morbidität des Todes mit Karzinom übersehen. Man stützt sich auf nicht validierte Annahmen.

\section{Punkte im Einzelnen}

Es ist wesentlich, die Begründungen der Boards nachzuvollziehen und die Datenlage vertieft zu verfolgen. Die Argumentation lautete:

1. The findings of the two largest trials highlight the uncertainty that remains about the precise effect that screening may have, and demonstrate that if any benefit does exist, it is very small after 10 years (...) (PLCO and ERSPC) $[1,2]$

2. The European trial found a statistically insignificant $0,06 \%$ reduction for men aged $50-74$ years, while the U.S. trial found a statistically insignificant $0,03 \%$ absolute increase in prostate cancer death $(. .$.

3. A meta-analysis of all published trials found no significant reduction in prostate cancer death (...) (Ilic BJUI 2011) [3]

4. Prostate cancer causes death in older ages.

Medical Board zusätzlich:

5. Eine Reihe von behandlungswürdigen Karzinomen wird vom PSA-Test übersehen.

6. Eine Kosten-Nutzen-Analyse zeigt, dass insgesamt mehr Kosten anfallen und weniger Wirkung erzielt wird, als wenn die PSA-Bestimmung unterlassen würde.

\section{Zu den Punkten 1 und 2}

Die Fehler beider Gruppen rühren vom fehlenden tieferen Verständnis des natürlichen Verlaufes der Erkrankung und der fehlenden vertieften Analyse der Screening-Studien. Es wird eine Beurteilung beinahe ausschliesslich anhand von vermeintlich validen 10-Jahres-Ergebnissen gefällt, eine Zeitspanne, die auch unter konservativer Therapie die meisten lokal begrenzten Tumoren erreichen.

Ausserdem darf der amerikanische PLCO-Trial nicht wie die ERSPC-Studie mit B1 bewertet werden, da er gravierende methodische Mängel aufweist, die es ihm verwehren, überhaupt eine Aussage über einen Screening Benefit zu treffen. (Vorscreening 44\%/fehlende Biopsien der PSA auffälligen Männer/ verspätete Aufnahme initial schwach rekrutierter Teilnehmer usw.). Die statistischen Voraussetzungen wurden im Studienverlauf in keiner Weise erfüllt und endeten in einer absoluten Unterpowerung (unverständlicherweise nicht dokumentiert oder erwähnt im NEJM 2009) [5]. Aufgedeckt wird all dies anhand der Prostatektomiepräparate. Man sollte erwarten, dass in der Screeninggruppe eine Stadienverschiebung in Richtung organbegrenzterer und weniger aggressiver Tumoren stattfindet. Dies fehlt beim PLCO Trial verglichen mit der ERSPC (T3: 6,2\% vs. 4,5\%, ERSPC: $20,5 \%$ vs. $9,5 \%$; high Gleason score $11,5 \%$ vs. $8,4 \%$;
ERSPC: $13,4 \%$ vs. 6,6\%). Salopp formuliert, sollte in Studien auch «Screening drin sein, wo Screening drauf steht».

\section{Zu Punkt 3}

Die U.S.PSTF und die Medical-Board-Aussagen basieren zudem auf der Cochrane Metaanalyse von Ilic (BJUI 2011). Aber: «Eine Aussage kann nur so gut sein wie das eingespiesene Datenmaterial.» Es werden die Daten von PLCO, ERSPC, Quebec, Norrköping u. Stockholm verwendet.

Die 1987 von Hausärzten initiierte Norrköping Studie ist sogar laut Autorenschaft «underpowered» und damit nicht aussagekräftig. Sie basiert zudem grösstenteils auf digital rektalen Untersuchungen (DRU) und soll die Frage PSA klären! Ausserdem erhielten nur $25 \%$ der Karzinomträger eine kurative Therapie Der Einschluss einer solchen Studie in die Metaanalyse kann keinen Beitrag zur Frage Screening Ja oder Nein leisten!

Auch in der Stockholm-Studie ist die nähere Analyse enttäuschend. Es wurden 62 von 65 Tumoren durch DRU entdeckt. PSA wurde nur rudimentär eingesetzt. Lediglich 11 von 65 Tumoren wurden überhaupt kurativ behandelt. Paradoxerweise zeigte die Kontrollgruppe mehr Prostatatumoren als die vermeintliche Screeninggruppe. Der Einschluss dieser Studien ist eher unglücklich. Die Quebec-Daten, die das Screening unterstützen, sind schon an anderer Stelle als nicht aussagekräftig analysiert worden $[6,7]$.

Die ERSPC-Ergebnisse einer Intention-To-TreatReduktion 20\% bzw. 27-30\% unter Ausschluss der Nicht-Teilnehmer im Screeningarm und der PSA-User im Kontrollarm nach 9 Jahren sind absolut vorläufig. Es ist für beide Boards zu frühzeitig, den Versuch einer Quantifizierung durchzuführen. Die Publikation der 11-Jahres-Daten der ERSPC steht bevor. Eine präzisere Beurteilung wird erst nach 12-14 Jahren erlaubt sein. Wesentlich ist, dass die Entwicklung in allen ERSPC-Zentren in die gleiche Richtung einer Mortalitätsreduktion durch Vorsorge verläuft. Die längsten Daten aus Göteborg (14 Jahre) dokumentieren mit ca. 50\% Mortalitätsreduktion einen Überlebensbenefit. Die NNS (Number Needed to Screen) 293, NNDiagnose 12, NNTreat 8 sind vergleichbar/ besser mit den Vorsorgeuntersuchungen anderen Tumorentitäten [8]. Ein Vergleich der jeweiligen PSA Cutoffs und Intervalle nach 12-14 Jahren wird interessant sein.

\section{Zu Punkt 4}

50-65jährige Männer haben noch eine Lebenserwartung von 20-32 Jahren. 4,5\% der Männer sterben in der Schweiz an einem Prostatakarzinom, ca. 14\% davon bis zum 69. Lebensjahr. Hier sind die SEER Krebsstatistiken mit dem auf PSA basierenden Rückgang der Sterblichkeit richtungsweisend [9]. Frau Etzoni hat anhand von zwei Modellen (des Fred Hutchinson Cancer Research Center und der University of Michi- 
gan) analysiert, dass 45-70\% der Mortalitätsreduktion auf den PSA-Test zurückzuführen ist [10]. Diesen Männern die Aufklärung und PSA zu verwehren, ist genauso einseitig wie die unkritische Anwendung von PSA.

\section{Zu Punkt 5}

Der Medical Board-Vorwurf, dass das PSA «eine Reihe von behandlungswürdigen Tumoren übersieht», beruht auf einer statischen bioptischen Studie von Thompson et al. ohne klinischen Follow-up als Beurteilungskriterium [11]. Die Schweizer Daten der ERSPC belegen das Gegenteil, unterhalb eines PSA $<3,0 \mathrm{ng} / \mathrm{ml}$ entwickeln nur 0,27\% (12/4332) der Männer innerhalb von 4 Jahren Follow-up (Intervalltumoren/2. Screening-Runde) einen High Risk Tumor. (4. Prostata Symposium St.Gallen 2011) [12 a, b]). Das heisst, der PSA-Test übersieht vernünftigerweise die Tumoren $<3,0 \mathrm{ng} / \mathrm{ml}$. Der Verlauf der minimst übersehenen Tumoren $(0,27 \%)$ in Bezug auf die Mortalität am Prostatakarzinom wird zu verfolgen sein, um festzustellen, welche Bedeutung dieser marginale Anteil hat.

\section{Zu Punkt 6}

Was den Kosten-Nutzen-Effekt des Screenings angeht, so ist selbst die WHO vor solchen Berechnungen zurückgeschreckt. Das methodisch schwierige Unterfangen basiert auf grösstenteils nicht validierten Annahmen. Neben vielen Unzulänglichkeiten (z.B. wird eine zweijährige Verminderung der Lebensqualität bei falsch-positiven PSA-Test-Ergebnissen angenommen) wird der Leidensweg der Gruppe von Männern komplett unterschlagen, die nicht am, sondern mit dem Karzinom sterben (palliative transurethrale Resektion der Prostata, Hormonentzug, Schmerzbehandlung usw.).

Es braucht den informierten Mann [13, 14], der nach eigener Beurteilung entscheidet, ob Vorsorge Ja oder Nein. Bei Bejahung der Vorsorge ist ein «intelligenter Einsatz des PSA» wesentlich. Der mit Schweizer Unterstützung entwickelt ERSPC Risk Calculator gibt das Risiko eines Prostatakarzinoms anhand IPSS (= International Prostata Symptom Score), Familienanamnese und PSA an, sowie, im Anschluss an eine etwaige positive Biopsie, die Wahrscheinlichkeit, einen klinisch nicht relevanten Tumor zu haben [15]. Die Anzahl der urologisch onkologischen Therapien muss durch Active Surveillance reduziert werden. Die Schweizerische Gesellschaft für Urologie hat seit geraumer Zeit eine landesweite Datenbank erstellt, in der alle Active-Surveillance-Patienten erfasst werden (SIPCAS).
Besonnen angewandt, ist der Test nach wie vor unverzichtbar. Man darf «das Kind nicht mit dem Bade ausschütten»!

\section{Literatur}

1 Andriole GL, Crawford ED, Grubb RL, 3rd, et al. Mortality results from a randomized prostate-cancer screening trial. N Engl J Med. 2009;360(13):1310-9.

2 Schroder FH, Hugosson J, Roobol MJ, et al. Screening and prostate-cancer mortality in a randomized European study. N Engl J Med. 2009;360(13):1320-8.

3 Ilic D, O'Connor D, Green S, Wilt TJ. Screening for prostate cancer: an updated Cochrane systematic review. BJU Int;107(6):882-91.

4 Prorok PC, Andriole GL, Bresalier RS, et al. Design of the Prostate, Lung, Colorectal and Ovarian (PLCO) Cancer Screening Trial. Control Clin Trials. 2000;21(6 Suppl):273S-309S.

5 Schröder FH. Stratifying risk - the U.S. Preventive services task force and prostate-cancer screening. N Engl J Med. 2011 Nov 24;365(21):1953-5. Epub 2011 Oct 26.

6 Boer R, Schroder FH. Quebec randomized controlled trial on prostate cancer screening shows no evidence for mortality reduction. Prostate. 1999,40(2):130-4.

7 Alexander FE, Prescott RJ. Reply to Labrie et al. Results of the mortality analysis of the Quebec randomized/controlled trial (RCT). Prostate. $1999,40(2) 135-7$

8 Hugosson J, Carlsson S, Aus G, et al. Mortality results from the Goteborg randomised populationbased prostate-cancer screening trial. Lancet Oncol.11(8):725-32.

9 Jemal A, Siegel R, Xu J, Ward E. Cancer statistics, 2010. CA Cancer J Clin;60(5):277-300.

10 Etzioni R, Tsodikov A, Mariotto A, et al. Quantifying the role of PSA screening in the US prostate cancer mortality decline. Cancer Causes Control. 2008;19(2):175-81

11 Thompson IM, Pauler DK, Coltman CA Jr.: Prevalence of prostate cancer among men with a prostate-specific antigen level $<$ or $=4.0 \mathrm{ng}$ per milliliter.

N Engl J Med. 2004 May 27;350(22):2239-46.

12 a www.informed-scientist.org/

12 b www.informed-scientist.org/presentation/ psa-screening-heute-nach-drei-grossen-randomisierten-studien

13 www.urologie.ch/v2/IMG/pdf/ informationen_zur_prostatakrebsvorsorge.pdf

14 www.urologie.ch/v2/IMG/pdf/information_an_ interessierte_prostatakrebs-vorsorge.pdf

15 www.prostatecancer-riskcalculator.com (ERSPC) 